\title{
Melting Phenomenon in a Squeezed Rheology of Reactive Rate Type Fluid
}

\author{
Shakeel Ahmad ${ }^{1 *}$, Muhammad Farooq ${ }^{1}$, Muhammad Rizwan ${ }^{2}$, Babar Ahmad ${ }^{3}$ and \\ Saif Ur Rehman ${ }^{3}$ \\ ${ }^{1}$ Department of Mathematics and Statistics, Riphah International University, Islamabad, Pakistan, ${ }^{2}$ Department of General \\ Studies, Jubail Industrial College, Jubail, Saudi Arabia, ${ }^{3}$ Department of Mathematics, COMSATS University, Islamabad, \\ Pakistan
}

The present study concentrates on squeezed unsteady magneto-hydrodynamic flow of Jeffrey fluid confined between two infinite parallel plates. Description of heat transfer process is disclosed through melting and thermal radiation effects. Features of viscous dissipation are also incorporated. Characteristics of mass transport are explored with chemical reaction. Modulated non-linear partial differential equations are reduced by implementing appropriate transformations. Approximate convergent solutions are calculated through analytical technique. Characteristics of velocity, concentration and fluid temperature are illustrated graphically and also discussed comprehensively physically. The skin friction co-efficient and Nusselt number are sketched and discussed through graphs. It is noticed that horizontal velocity component and temperature of Jeffrey liquid are dominant for greater melting parameter. Moreover, temperature field

OPEN ACCESS

Edited by:

Dumitru Baleanu,

University of Craiova, Romania

Reviewed by:

Amin Jajarmi,

University of Bojnord, Iran

Kazuharu Bamba,

Fukushima University, Japan

*Correspondence:

Shakeel Ahmad

shakeel_oiiui@hotmail.com

Specialty section:

This article was submitted to

Mathematical Physics,

a section of the journal

Frontiers in Physics

Received: 18 November 2019

Accepted: 23 March 2020

Published: 06 May 2020

Citation:

Ahmad S, Farooq M, Rizwan M

Ahmad B and Rehman SU (2020)

Melting Phenomenon in a Squeezed

Rheology of Reactive Rate Type Fluid.

Front. Phys. 8:108.

doi: 10.3389/fphy.2020.00108 decays for dominant thermal radiation parameter.

Keywords: jeffrey fluid, squeezing flow, melting heat transfer, viscous dissipation, thermal radiation, chemical reaction

\section{INTRODUCTION}

Recently, scientists and researchers have shown intent to analyze the features of non-Newtonian fluid flow imposed by squeezed surfaces, regarding their wide-spread build up and trade area in many industrial and biological processes, such as in the polymer industry, compression, injection shaping, liquid-metal lubrication, formation of paper sheets and thin fiber, molding of plastic sheets and metal and squeezed film, through which power is transmitted. Many researchers discussed the Jeffrey fluid model corresponding to various physical aspects. But this model is not studied widely with the squeezing phenomenon. At beginning, Stefan [1] depicted work on squeezing flow. Muhammad et al. [2] exposed squeezed nanomaterial flow with modified version of heat and mass fluxes. Adesanya et al. [3] showed the radiative and chemically reactive flow of Powell-Eyring liquid squeezed through infinite parallel walls. MHD-squeezed fluid flow confined between parallel two plates under suction/injection has been constructed by Su and Yin [4]. Naduvinamani and Shankar [5] disclosed the heat transport phenomenon due to radiative MHD-squeezed flow in a Casson fluid. Ullah et al. [6] exhibited squeezed flows through porous medium under aspect of magnetic field strength. Lahmar et al. [7] demonstrated the characteristics of the heat transport phenomenon due to varying thermal conductivity on MHD-squeezed nanoparticle. Khan et al. [8] analyzed entropy generation features in autocatalytic-squeezed flow through parallel sheets analytically.

Characteristics of heat transfer have increased noteworthy interest of recent explorers and engineers because of various technical processes in the area of engineering, physiological and industrial processes, including glass blowing and glass fiber productions, wire coating, paper making, metal spinning, expulsion of plastic sheets by aerodynamic, drawing of plastic films and 
unceasing casting. However, little attention is paid to disclose the features of heat transport through melting phenomenon. It has widespread applications, such as the making of semiconductors, melting permafrost and hardening of magma flow, heat exchanger coils coated by freeze soil in grounded pump, sewage treatment via freeze process, melting of soil, welding processes, and casting of manufacturing processes. Behavior of doublediffusive convection in stagnant Maxwell liquid flow considering melting phenomenon has been explored by Hayat et al. [9]. Krishnamurthy et al. [10] scrutinized the Williamson nanofluid flow via porous structure. Behavior of Carreau liquid flow caused by stretchable cylinder considering melting phenomenon has been illustrated by Hashim et al. [11]. Soomro et al. [12] explored the melting effects in radiative Sisko fluid flow past a moving sheet. Mabood et al. [13] depicted the aspects of heat transport through melting condition in radiative Sisko nanofluid flowing over stretchable sheet with heat generation/absorption. Javed et al. [14] reported the domination of heat transport phenomenon via melting affects the MHD reactive liquid flow through non-porous stretchable surfaces of varying thickness. Hayat et al. [15] exhibited the features of melting flow phenomenon in the squeezed third grade liquid flow past a plate with modified heat flux phenomenon. Irreversibility and melting heat transport properties in dissipative flow of liquid passed through a stretched sheet has been exhibited by Hayat et al. [16]. Properties of melting flow phenomenon in hydromagnetic nanomaterial flow are depicted by Dinh et al. [17]. Their investigations motivated a few other researchers to work on heat transport in different conditions $[18,19]$.

A literature survey indicates that researchers have disclosed the properties of squeezed Newtonian and non-Newtonian fluid flow with different boundary conditions of heat transfer widely. But such analysis of squeezing flow with melting heat transport has not explored as of yet. So, the main strategic concern is to fill this void. Thus, the present attempt is concerned to demonstrate the melting mechanism in squeezed Jeffrey fluid flow through two parallel plates. Behavior of heat with mass transport is modeled and exposed considering thermal radiation, viscous dissipation and chemical reaction. The transformed coupled non-linear differential system is evaluated via a homotopy technique [20-24]. Graphical studied is evaluated for the features of velocity, liquid temperature and fluid concentration via emerging parameters. Moreover, the layout of the current paper is as follows. Mathematical formulation describes the mathematical modeling of governing equations related to the work under consideration. Homotopy analysis solutions illustrates the solution method utilized for the flow analysis. The acquired results are discussed and analyzed in Analysis. Closing remarks is related to key points of present paper.

\section{MATHEMATICAL FORMULATION}

Consider unsteady, incompressible squeezed Jeffrey fluid flow between horizontal two plates. The bottom plate is located at $y=$ 0 while top sheet is placed at $y=h(t)=\sqrt{v(1-\gamma t) / a}$, which approaches stretchable bottom plate with squeeze velocity. Fluid is assumed to be electrically conducting so that fluid is influenced by applied magnetic field along vertical direction. The induced magnetic and electric fields are neglected, considering the small Reynolds number (magnetic). Heat transport phenomenon is characterized by a modern and advanced condition at boundary (i.e., melting heat transport). Viscous dissipation and thermal radiation are assumed to elaborate the heat transfer phenomenon. Features of mass transfer are explored via first order chemical reaction. We consider Cartesian co-ordinates $(x, y)$ and physical situation to be exposed in Figure 1. The flow problem under the basic conservative laws takes the form:

$$
\begin{aligned}
& \frac{\partial u}{\partial x}+\frac{\partial v}{\partial y}=0 \\
& \rho\left(\frac{\partial u}{\partial t}+u \frac{\partial u}{\partial x}+v \frac{\partial u}{\partial y}\right)=-\frac{\partial p}{\partial x}+\frac{\mu}{1+\lambda_{1}}\left(\frac{\partial^{2} u}{\partial x^{2}}+\frac{\partial^{2} u}{\partial y^{2}}\right. \\
& +\lambda_{2}\left(\frac{\partial^{3} u}{\partial x^{2} \partial t}+u \frac{\partial^{3} u}{\partial x^{3}}+v \frac{\partial^{3} u}{\partial x^{2} \partial y}+2 \frac{\partial u}{\partial x} \frac{\partial^{2} u}{\partial x^{2}}+2 \frac{\partial v}{\partial x} \frac{\partial^{2} u}{\partial x \partial y}\right. \\
& +\frac{\partial^{3} u}{\partial y^{2} \partial t}+\frac{\partial u}{\partial y} \frac{\partial^{2} u}{\partial y \partial x}+u \frac{\partial^{3} u}{\partial y^{2} \partial x}+\frac{\partial v}{\partial y} \frac{\partial^{2} u}{\partial y^{2}}+v \frac{\partial^{3} u}{\partial y^{3}}+\frac{\partial u}{\partial y} \frac{\partial^{2} v}{\partial x^{2}} \\
& \left.\left.+\frac{\partial v}{\partial y} \frac{\partial^{2} v}{\partial x \partial y}\right)\right)-\frac{\sigma_{1} B_{0}^{2}}{1-\gamma t} u \\
& \rho\left(\frac{\partial v}{\partial t}+u \frac{\partial v}{\partial x}+v \frac{\partial v}{\partial y}\right)=-\frac{\partial p}{\partial y}+\frac{\mu}{1+\lambda_{1}}\left(\frac{\partial^{2} v}{\partial x^{2}}+\frac{\partial^{2} v}{\partial y^{2}}\right. \\
& +\lambda_{2}\left(\frac{\partial^{3} v}{\partial x^{2} \partial t}+u \frac{\partial^{3} v}{\partial x^{3}}+v \frac{\partial^{3} v}{\partial x^{2} \partial y}+2 \frac{\partial v}{\partial y} \frac{\partial^{2} v}{\partial y^{2}}+2 \frac{\partial u}{\partial y} \frac{\partial^{2} v}{\partial x \partial y}\right. \\
& +\frac{\partial^{3} v}{\partial y^{2} \partial t}+\frac{\partial u}{\partial x} \frac{\partial^{2} u}{\partial y \partial x}+u \frac{\partial^{3} v}{\partial y^{2} \partial x}+\frac{\partial v}{\partial x} \frac{\partial^{2} u}{\partial y^{2}}+v \frac{\partial^{3} v}{\partial y^{3}}+\frac{\partial u}{\partial x} \frac{\partial^{2} v}{\partial x^{2}} \\
& \left.\left.+\frac{\partial v}{\partial x} \frac{\partial^{2} v}{\partial x \partial y}\right)\right) \\
& \rho c_{p}\left(\frac{\partial T}{\partial t}+u \frac{\partial T}{\partial x}+v \frac{\partial T}{\partial y}\right)=k_{2}\left(\frac{\partial^{2} T}{\partial x^{2}}+\frac{\partial^{2} T}{\partial y^{2}}\right) \\
& +\frac{16 \sigma^{*} T_{\infty}^{3}}{3 k_{1}} \frac{\partial^{2} T}{\partial y^{2}}+\frac{\mu}{1+\lambda_{1}}\left(2\left(\frac{\partial u}{\partial x}\right)^{2}+2\left(\frac{\partial v}{\partial y}\right)^{2}\right. \\
& +\left(\frac{\partial u}{\partial y}+\frac{\partial v}{\partial x}\right)^{2}+\lambda_{2}\left(2 \frac{\partial u}{\partial x}\left(\frac{\partial^{2} u}{\partial x \partial t}+u \frac{\partial^{2} u}{\partial x^{2}}+v \frac{\partial^{2} u}{\partial x \partial y}\right)\right. \\
& +2 \frac{\partial v}{\partial y}\left(\frac{\partial^{2} v}{\partial y \partial t}+v \frac{\partial^{2} v}{\partial y^{2}}+u \frac{\partial^{2} v}{\partial x \partial y}\right)+\left(\frac{\partial u}{\partial y}+\frac{\partial v}{\partial x}\right)\left(\frac{\partial^{2} u}{\partial y \partial t}\right. \\
& \left.\left.\left.+\frac{\partial^{2} v}{\partial x \partial t}+u \frac{\partial^{2} u}{\partial x \partial y}+v \frac{\partial^{2} u}{\partial y^{2}}+u \frac{\partial^{2} v}{\partial x^{2}}+v \frac{\partial^{2} v}{\partial x \partial y}\right)\right)\right), \\
& \left(\frac{\partial C}{\partial t}+u \frac{\partial C}{\partial x}+v \frac{\partial C}{\partial y}\right)=D\left(\frac{\partial^{2} C}{\partial x^{2}}+\frac{\partial^{2} C}{\partial y^{2}}\right)-K(t) C .
\end{aligned}
$$

Here, velocity components are $u$ and $v$ along $x$ and $y$ direction, respectively. $p$ represents pressure, $\mu$ represents absolute viscosity, $\gamma$ is the dimensional positive constant, $B_{0}$ represents magnetic field, $T$ denotes the fluid temperature, $\rho$ represents the fluid density, $\sigma_{1}$ denotes electric conductivity, $C$ denotes fluid concentration, $k_{2}$ is the co-efficient of thermal conductivity, specific heat is $c_{p}, k_{1}$ is absorption coefficient, $\sigma^{*}$ 


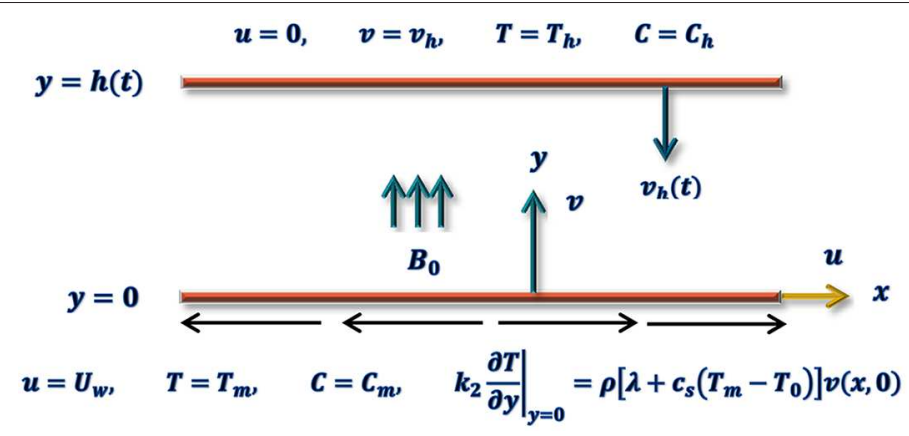

FIGURE 1 | Physical situation of the problem.

denotes the Stefan-Boltzmann constant, $D$ is the diffusion coefficient, $v$ is the kinematics viscosity, $K(t)=K_{0} / 1-\gamma t$ is timedependent reaction rate with reaction rate $K_{0}, \lambda_{1}$ is relaxation time and $\lambda_{2}$ is ratio among relaxation and retardation time.

The boundary conditions are as follow

$$
\begin{aligned}
u & =U_{w}=\frac{a x}{1-\gamma t}, T=T_{m}, \quad C=C_{m}, \quad \text { at } y=0, \\
u & =0, v=v_{h}=\frac{d h}{d t}=-\frac{\gamma}{2} \sqrt{\frac{v}{a(1-\gamma t)}}, T=T_{h}, \\
C & =C_{h} \quad \text { at } y=h(t), \\
\left.k_{2} \frac{\partial T}{\partial y}\right|_{y=0} & =\rho\left[\lambda+c_{s}\left(T_{m}-T_{0}\right)\right] v(x, 0) .
\end{aligned}
$$

Transformations are taken as follows

$$
\begin{aligned}
\eta & =\frac{y}{h(t)}, \Psi=\sqrt{\frac{a \nu}{1-\gamma t}} x f(\eta), \quad u=U_{w} f^{\prime}(\eta), \\
v & =-\sqrt{\frac{a \nu}{1-\gamma t}} f(\eta), \\
\theta(\eta) & =\frac{T-T_{m}}{T_{h}-T_{m}}, \quad \phi(\eta)=\frac{C-C_{m}}{C_{h}-C_{m}} .
\end{aligned}
$$

Pressure term is eliminated from equations (2) and (3), governing equations in view of equation (7) take the forms:

$$
\begin{aligned}
& f^{(i v)}+\left(1+\lambda_{1}\right)\left(f^{\prime \prime \prime}-f^{\prime} f^{\prime \prime}-\frac{S_{q}}{2}\left(3 f^{\prime \prime}+\eta f^{\prime \prime \prime}\right)\right) \\
& +\beta\left(\frac{S_{q}}{2}\left(5 f^{(i v)}+\eta f^{(v)}\right)+2 f^{\prime \prime} f^{\prime \prime \prime}-f^{\prime} f^{(i v)}-f^{(v)}\right) \\
& -H a^{2} f^{\prime \prime}=0, \\
& \left(1+\lambda_{1}\right)\left(1+N_{r}\right) \theta^{\prime \prime}+\operatorname{Pr}\left(1+\lambda_{1}\right)\left(f \theta^{\prime}-\frac{S_{q}}{2} \eta \theta^{\prime}\right) \\
& +\operatorname{PrEc}\left(\left(f^{\prime \prime}\right)^{2}+4 \delta^{2}\left(f^{\prime}\right)^{2}+\beta\left(4 \delta ^ { 2 } \left(\frac{S_{q}}{2}\left(2\left(f^{\prime}\right)^{2}+\eta f^{\prime} f^{\prime \prime}\right)\right.\right.\right. \\
& \left.-f^{\prime} f^{\prime \prime}\right)+\left(\frac{S_{q}}{2}\left(3\left(f^{\prime \prime}\right)^{2}+\eta f^{\prime \prime} f^{\prime \prime \prime}\right)+f^{\prime}\left(f^{\prime \prime}\right)^{2}\right. \\
& \left.\left.\left.-f^{\prime \prime} f^{\prime \prime \prime}\right)\right)\right)=0, \\
& \phi^{\prime \prime}-S c\left(\frac{S_{q}}{2} \eta \phi^{\prime}-f \phi^{\prime}\right)-S c \sigma \phi=0 .
\end{aligned}
$$

The subjected boundary conditions are

$$
\begin{aligned}
& f(1)=\frac{S_{q}}{2}, \quad f^{\prime}(0)=1, f^{\prime}(1)=0, M \theta^{\prime}(0)+\operatorname{Prf}(0)=0, \\
& \theta(0)=0, \quad \theta(1)=1, \phi(0)=0, \phi(1)=1,
\end{aligned}
$$

where squeezing parameter is $S_{q}$, Deborah number is $\beta$, magnetic parameter is $\mathrm{Ha}$, Prandtl number is $\mathrm{Pr}$, Schmidt number is $\mathrm{Sc}$, thermal radiation parameter is $N_{r}$, Eckert number is $E c$, the chemical reaction parameter is $\sigma$ and the melting parameter is $M$.

These dimensionless quantities are given by

$$
\begin{aligned}
& S_{q}=\frac{\gamma}{a}, \beta=\frac{a \lambda_{2}}{1-\gamma t}, H a^{2}=\frac{\sigma_{1} B_{0}^{2}}{\rho a}, N_{r}=\frac{16 \sigma^{*} T_{\infty}^{3}}{3 k_{1} k_{2}}, \\
& \operatorname{Pr}=\frac{\mu c_{p}}{k_{2}}, S c=\frac{v}{D} \\
& E c=\frac{a^{2} x^{2}}{c_{p}(1-\gamma t)^{2}\left(T_{h}-T_{m}\right)}, \quad \sigma=\frac{K_{o}}{a} \\
& M=\frac{c_{p}\left(T_{h}-T_{m}\right)}{\left[\lambda+c_{s}\left(T_{m}-T_{0}\right)\right]}, \delta=\sqrt{\frac{\nu(1-\gamma t)}{a x^{2}}}
\end{aligned}
$$

Viscous dissipation effect vanishes when $E c=0$. Further, $\sigma>0$ gives destructive and $\sigma<0$ gives generative chemical reactions. When $\beta=0, \lambda_{1}=0$, Jeffrey fluid converts to viscous fluid. Moreover, the plates illustrate inward movement for $S_{q}>0$, and for $S_{q}<0$, away movement is observed.

Defining skin friction co-efficient, heat and mass transfer rate as follows

$$
\begin{aligned}
C f & =\frac{\left.\mu\left(\tau_{x y}\right)\right|_{y=h(t)}}{\rho U_{w}^{2}}, N u=\frac{-\left.x k_{2}\left(1+\frac{16 \sigma^{*} T_{\infty}^{3}}{3 k_{1} k_{2}}\right)\left(\frac{\partial T}{\partial y}\right)\right|_{y=h(t)}}{k_{2}\left(T_{h}-T_{m}\right)} \\
S h & =\frac{-\left.x D\left(\frac{\partial C}{\partial y}\right)\right|_{y=h(t)}}{D\left(C_{h}-C_{m}\right)}
\end{aligned}
$$

In view of dimensionless forms, one may write.

$$
\begin{aligned}
(R e)^{\frac{1}{2}} C f & =\frac{1}{1+\lambda_{1}}\left(f^{\prime \prime}(1)+\frac{3}{2} \beta S_{q} f^{\prime \prime}(1)\right) \\
(R e)^{-\frac{1}{2}} N u & =-\left(1+N_{r}\right) \theta^{\prime}(1),(R e)^{-\frac{1}{2}} S h=-\phi^{\prime}
\end{aligned}
$$




\section{HOMOTOPY ANALYSIS SOLUTIONS}

The homotopic procedure was initially purposed by Liao [20], which has been employed to construct the solutions of coupled non-linear problem. For this method, initial guesses $\left(f_{0}, g_{0}, j_{0}\right)$ and concerned linear operators $\mathcal{L}_{f}, \mathcal{L}_{\theta}, \mathcal{L}_{\Phi}$ for constitutive equations are defined in the form:

$$
\begin{aligned}
f_{0}(\eta) & =\frac{1}{2 P r}\left(-2 M+2 P r \eta+6 M \eta^{2}\right. \\
& \left.-4 P r \eta^{2}+3 P r S_{q} \eta^{2}-4 M \eta^{3}+2 P r \eta^{3}-2 \operatorname{Pr} S_{q} \eta^{3}\right) \\
\theta_{0}(\eta) & =\eta, \phi_{0}(\eta)=\eta, \\
\mathcal{L}_{f} & =f^{(i v)}, \quad \mathcal{L}_{\theta}=\theta^{\prime \prime}, \quad \mathcal{L}_{\phi}=\phi^{\prime \prime}
\end{aligned}
$$

with

$$
\begin{aligned}
& \mathcal{L}_{f}\left(C_{1}+C_{2} \eta+C_{3} \eta^{2}+C_{4} \eta^{3}\right)=0, \quad \mathcal{L}_{\theta}\left(C_{5}+C_{6} \eta\right)=0 \\
& \mathcal{L}_{\phi}\left(C_{7}+C_{8} \eta\right)=0 .
\end{aligned}
$$

where $C_{i}(i=1,2, \ldots .8)$ are arbitrary constants.

\section{Convergence of Problem}

Clearly, convergent homotopic solutions incorporate the auxiliary non-zero parameters $\hbar_{f}, \hbar_{\theta}$ and $\hbar_{\phi}$. Said parameters significantly provide way to regulate the convergence. In order to prefer the reasonable estimation of $\hbar_{f}, \hbar_{\theta}$ and $\hbar_{\phi}$, the $h$-curves are exhibited in Figure 2. It is pointed out that sufficient ranges for these parameters, namely $\hbar_{f}, \hbar_{\theta}$ and $\hbar_{\phi}$, are $-1.7 \leq \hbar_{f} \leq-0.1$ and $-1.7 \leq \hbar_{\theta} \leq 0.0$ and $-1.8 \leq \hbar_{\phi} \leq-0.1$.

\section{ANALYSIS}

The main attraction here is to discuss the stimulus of emerging parameters in flow fields (i.e., velocity, temperature, concentration) under consideration. Figure 3 represents the impact of squeeze parameter $\left(S_{q}\right)$ on the vertical and horizontal velocity fields. It is seen that increase in $S_{q}$ guarantees an increase in both velocity distributions, respectively. Physically, it is justified that higher squeezing parameter corresponded to larger deformation in fluid particles. Hence, the growing behavior of velocity is noted. Figure 4 elucidates the impacts of the ratio between relaxation and retardation time parameter

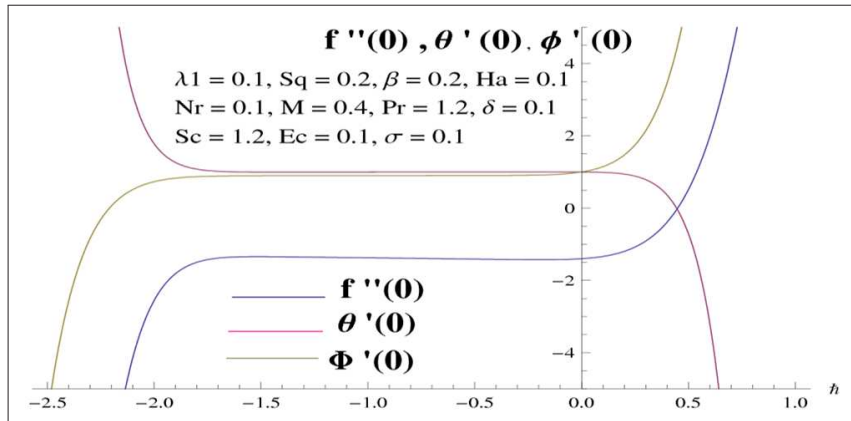

FIGURE 2 | Regions of convergence for $f(\eta), \theta(\eta)$, and $\phi(\eta)$. $\lambda_{1}$ on the horizontal component of velocity. It is delineated that horizontal velocity component decays for growing $\lambda_{1}$. Physically, greater values of $\lambda_{1}$ correspond to higher relaxation time. So, perturbed fluids require more time to take their original shape. Therefore, the velocity profile decreased. The
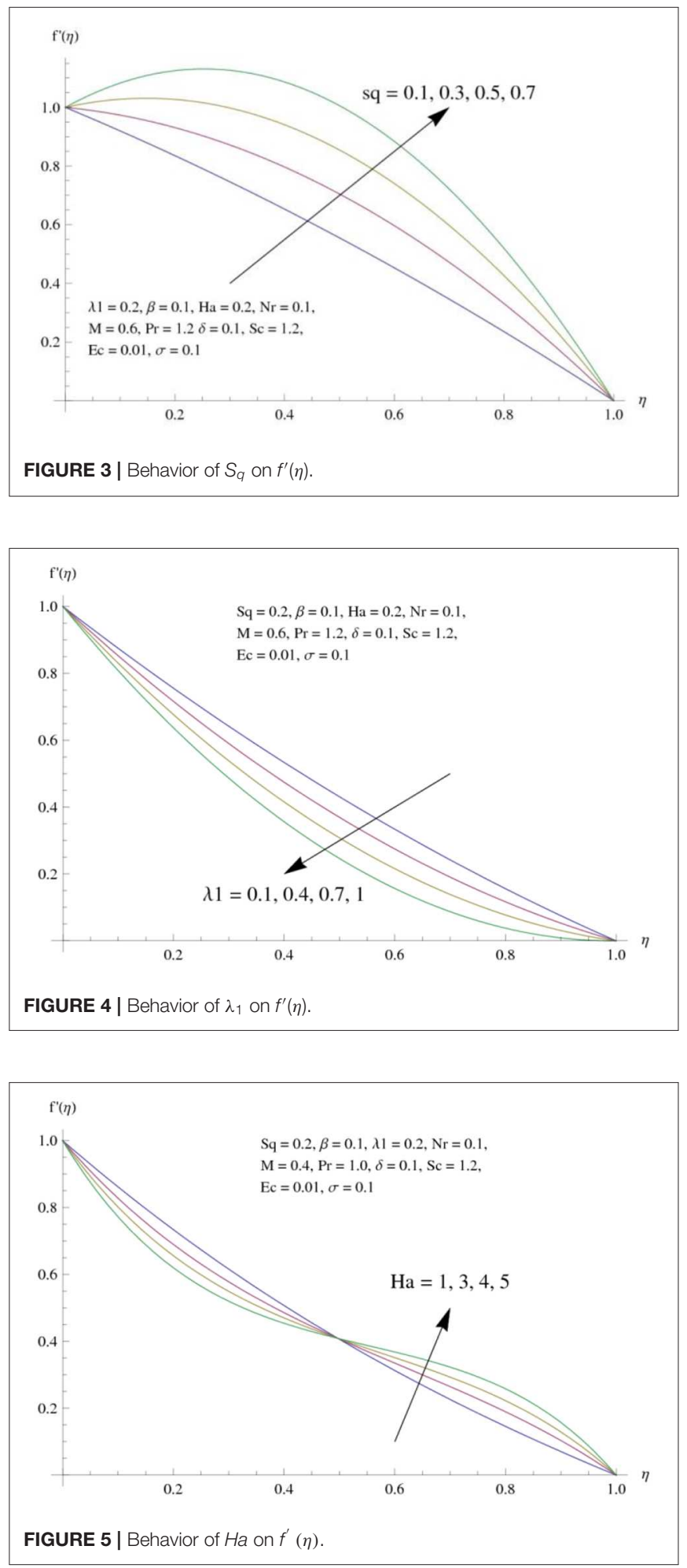
variation in velocity distribution for diverse values of the Hartman number is exhibited in Figure 5. The velocity of the consider liquid exhibits decreasing trend in a region $0 \leq$ $\eta \leq 0.5$, whereas it shows opposite behavior in the region $0.5 \leq \eta \leq 1$. Further, no variation is noticed in the fluid
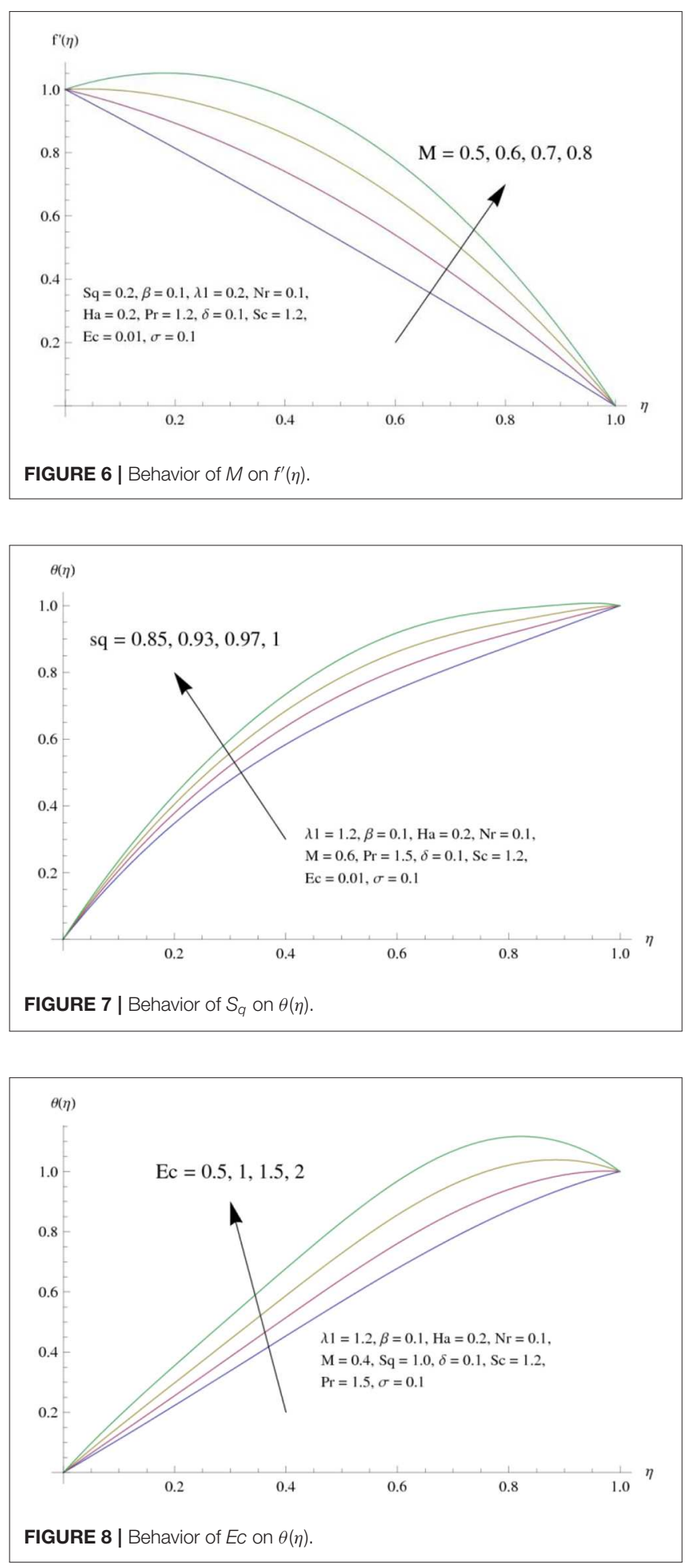

velocity at $\eta=0.5$. Larger Hartman number values correspond to more intensity of the Lorentz force, resulting resistance in fluid's motion. Hence, velocity distribution decays. Figure 6 represents the behavior of horizontal velocity for varying melting parameter. It is evident that horizontal velocity enhances in
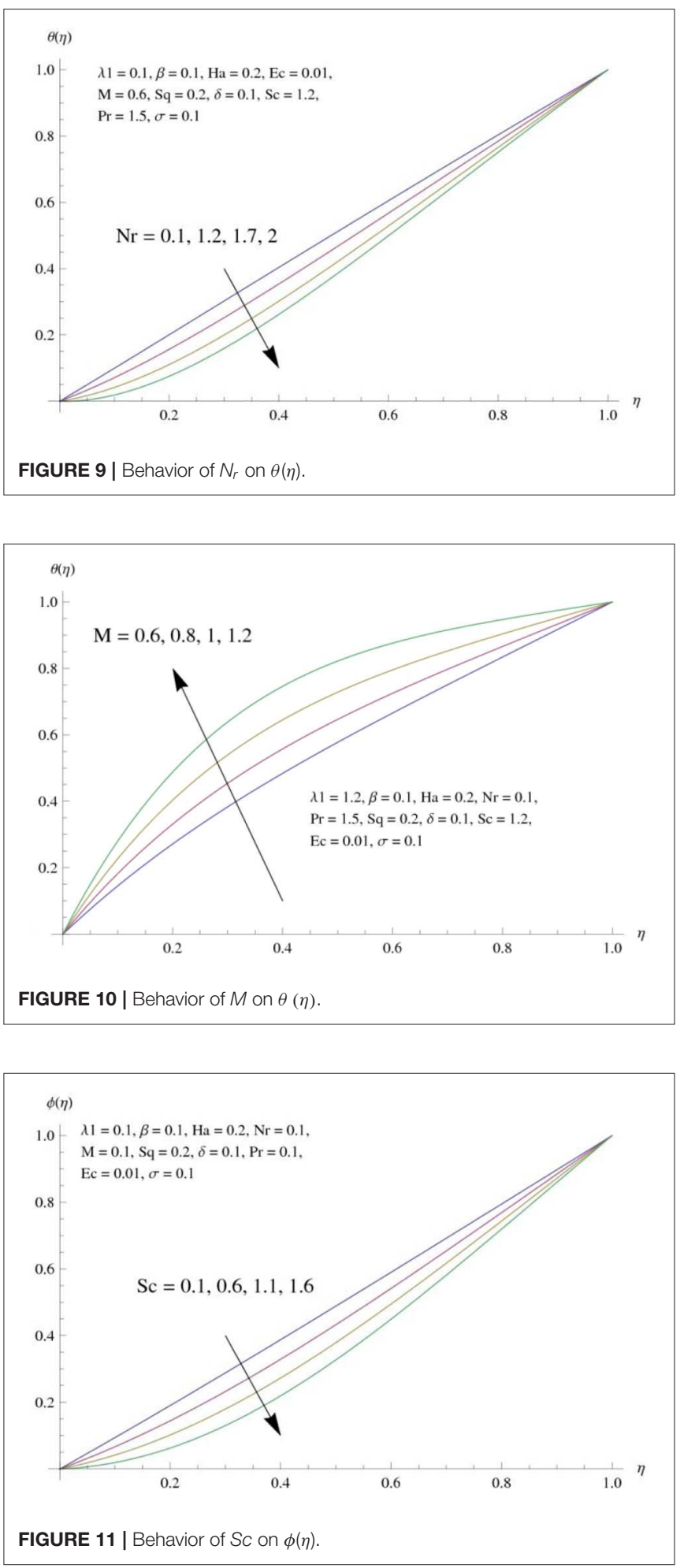


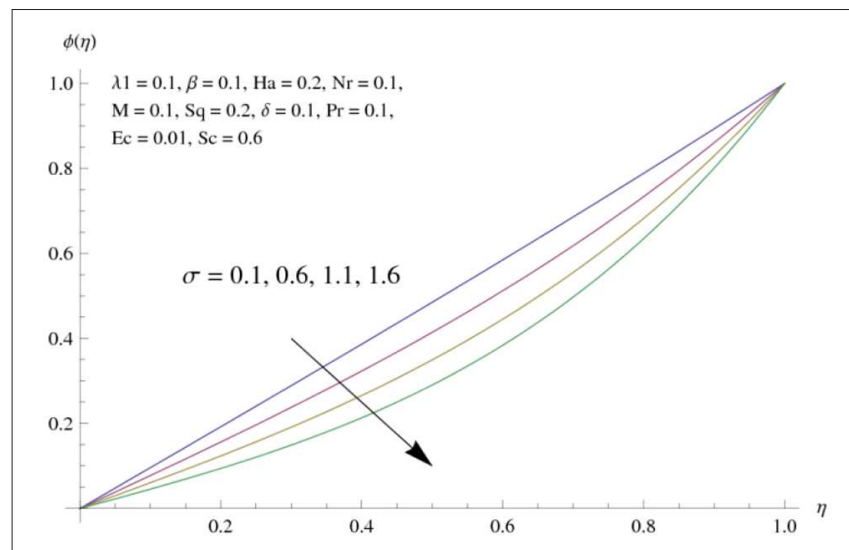

FIGURE 12 | Behavior of $\sigma$ on $\phi(\eta)$.
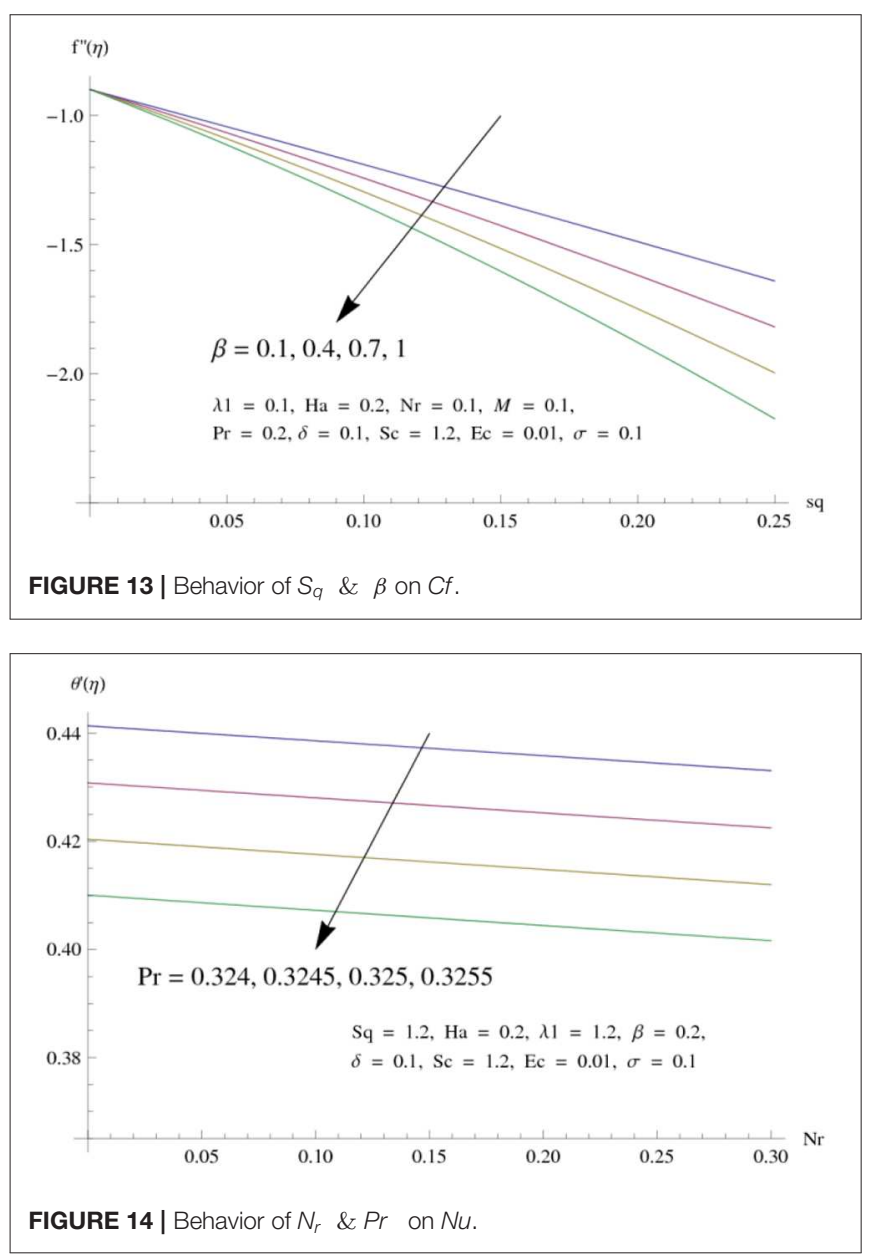

increments in the melting parameter. A physically enlarged melting parameter is responsible for a more convective flow in the transaction of fluid, which is heated toward the cold plate surface, and as a consequence, horizontal velocity component increases. Analysis of the temperature field against the squeezing parameter is depicted in Figure 7. Here, the temperature field becomes dominant for greater values of the squeezing parameter.
TABLE 1 | Comparison of skin friction co-efficient (Cf) for different values of $\mathrm{Sq}, \mathrm{Ha}, \quad \beta$, and $\lambda_{1}$.

\begin{tabular}{|c|c|c|c|c|c|}
\hline \multirow[b]{2}{*}{$S q$} & \multirow[b]{2}{*}{ Ha } & \multirow[b]{2}{*}{$\beta$} & \multirow[b]{2}{*}{$\lambda$} & \multirow{2}{*}{$\begin{array}{c}\text { Muhammad et al. [22] } \\
(R e)^{1 / 2} \mathrm{Cf}\end{array}$} & \multirow{2}{*}{$\frac{\text { Present }}{(R e)^{1 / 2} C f}$} \\
\hline & & & & & \\
\hline 0.0 & 1.0 & 0.1 & 0.5 & 4.646286 & 4.646286 \\
\hline 0.5 & & & & 3.513473 & 3.513473 \\
\hline 0.1 & & & & 2.059355 & 2.059355 \\
\hline \multirow[t]{3}{*}{1.0} & 0.0 & 0.1 & 0.5 & 2.079543 & 2.079543 \\
\hline & 0.5 & & & 2.074376 & 2.074376 \\
\hline & 1.0 & & & 2.059355 & 2.059355 \\
\hline \multirow[t]{3}{*}{1.0} & 1.0 & 0.0 & 0.5 & 1.697800 & 1.697800 \\
\hline & & 0.5 & & 2.059355 & 2.059355 \\
\hline & & 0.2 & & 2.393890 & 2.393890 \\
\hline \multirow[t]{3}{*}{1.0} & 1.0 & 0.1 & 0.0 & 2.133399 & 2.133399 \\
\hline & & & 0.5 & 2.059355 & 2.059355 \\
\hline & & & 1.0 & 1.990723 & 1.990723 \\
\hline
\end{tabular}

Further, higher temperature exists near the upper wall. In fact, the higher squeezing parameter corresponds to a greater squeeze force on the fluid, which leads to an increase in temperature profile. To capture the effects of the Eckert number on temperature distribution, Figure 8 is portrayed. With an increase in Eckert number, temperature field grows. Higher Eckert number is corresponding to higher friction force between the fluid particles which give rise to more heat generation. Hence liquid temperature amplifies. Description of thermal radiative parameter on liquid temperature is analyzed through Figure 9. Temperature field grows up for smaller thermal radiation parameter. Moreover, it is revealed that intensifies temperature occurs about upper plate surface. Physically, higher radiation parameter corresponds to lower absorption coefficient of the material. Thus, cold surface enhances more heat from heated fluid. Hence temperature field declines. Figure $\mathbf{1 0}$ addresses the variations of melting parameter in temperature field. It is reflected that temperature field goes up for higher melting parameter. Figure 11 exhibits the physical features of Schmidt number $(S c)$ on concentration curve. Decline is seen in concentration field with an increasing Schmidt number, which relates to momentum diffusivity and mass diffusivity. Thus, mass diffusivity diminishes for higher Schmidt number. Hence, liquid concentration weakens. Figure 12 exhibits the features of chemical reaction (destructive) parameter in concentration field. It demonstrates that higher chemical reaction parameter decays the concentration field. Figure $\mathbf{1 3}$ discloses the properties of squeezing parameter and the Deborah number on the skin friction. Skin friction intensifies for growing $S_{q}$ and $\beta$. Influences of $\operatorname{Pr}$ and $N_{r}$ on the Nussetl number are illustrated in Figure 14. It is seen that the Nussetl number weakens for greater Prandtl number and the thermal radiation parameter. To validate the current results, Table 1 displays a comparison of different values of skin friction with previously published work of Muhammad [22] for diverse values of $S_{q}, H a, \beta$ and $\lambda_{1}$. It is revealed that the outcome gives favorable agreement. 


\section{CLOSING REMARKS}

Here, we have investigated heat and mass transport phenomenon effects on squeezed Jeffrey liquid flow between two parallel walls. Heat and mass transport are featured with melting heat transport and first order chemical reaction, respectively. The conclusions are as follows:

- Diverse values of relaxation to retardation parameter $\lambda_{1}$ decay the horizontal velocity profile.

- Higher values of melting parameter $M$ enhance the horizontal velocity but vertical velocity shows decreasing behavior.

- Enhancing values of Eckert number $E c$ and melting parameter $M$ lead to in the enhancement in temperature field.

- Concentration distribution shows decreasing behavior with increment in chemical reaction parameter $\sigma$.

\section{REFERENCES}

1. Stefen MJ. Versuch Uber die scheinbare adhesion. Sitzungsberichte der Akademie der Wissenschaften in Wien. Mathematik-Naturwissen. (1874) 69:713-21.

2. Muhammad N, Nadeem S, Mustafa T. Squeezed flow of a nanofluid with Cattaneo-Christov heat and mass fluxes. Results Phys. (2017) 7:8629. doi: 10.1016/j.rinp.2016.12.028

3. Adesanya SO, Ogunseye HA, Jangili S. Unsteady squeezing flow of a radiative Eyring-Powell fluid channel flow with chemical reactions. Int J Therm Sci. (2018) 125:440-7. doi: 10.1016/j.ijthermalsci.2017.12.013

4. Su X, Yin Y. Effects of an inclined magnetic field on the unsteady squeezing flow between parallel plates with suction/injection. J Magn Magn Mater. (2019) 484:266-71. doi: 10.1016/j.jmmm.2019.04.041

5. Naduvinamani NB, Shankar U. Radiative squeezing flow of unsteady magneto-hydrodynamic Casson fluid between two parallel plates. J Cent South Univ. (2019) 26:1184-204. doi: 10.1007/s11771-0194080-0

6. Ullah I, Rahim MT, Khan H, Qayyum M. Analysis of various semi-numerical schemes for magnetohydrodynamic (MHD) squeezing fluid flow in porous medium. Propul Power Res. (2019) 8:69-78. doi: 10.1016/j.jppr.2019.01.003

7. Lahmar S, Kezzar M, Eid MR, Sari MR. Heat transfer of squeezing unsteady nanofluid flow under the effects of an inclined magnetic field and variable thermal conductivity. Physica A. (2020) 540:e123138. doi: 10.1016/j.physa.2019.123138

8. Khan MI, ur Rahman M, Khan SA, Hayat T, Khan MI. Evaluation of entropy generation in cubic autocatalytic unsteady squeezing flow of nanofluid between two parallel plates. Comp Meth Prog Biomed. (2020) 185:e105149. doi: 10.1016/j.cmpb.2019.105149

9. Hayat T, Farooq M, Alsaedi A. Melting heat transfer in the stagnation point flow of Maxwell fluid with double diffusive convection. Int J Num Meth Heat Fluid Flow. (2014) 24:760-74. doi: 10.1108/HFF-09-2012-0219

10. Krishnamurthy MR, Prasannakumara BC, Gireesha BJ, Gorla RSR. Effect of chemical reaction on MHD boundary layer flow and melting heat transfer of Williamson nanofluid in porous medium. Eng Sci Tech Int J. (2016) 19:53-61. doi: 10.1016/j.jestch.2015.06.010

11. Hashim, Khan M, Alshomrani AS. Characteristics of melting heat transfer during flow of Carreau fluid induced by a stretching cylinder. Eur Phys J Plus. (2017) 40:8. doi: 10.1140/epje/i2017-11495-6

12. Soomro FA, Usman M, Haq R, Wang W. Melting heat transfer analysis of Sisko fluid over a moving surface with nonlinear thermal radiation via Collocation method. J Heat Mass Trans. (2018) 126:1034-42. doi: 10.1016/j.ijheatmasstransfer.2018.05.099
It is expected that the present attempt relates the physical modeling under different flow conditions. It includes dynamical systems, lubrication mechanism, heating, cooling and energy estimation processes, etc. Further, as a future direction, the current investigation can tackle the different dynamical system [25-29].

\section{DATA AVAILABILITY STATEMENT}

All datasets generated for this study are included in the article/supplementary material.

\section{AUTHOR CONTRIBUTIONS}

SA collected the data and wrote the paper. MF made the analysis of the paper. $\mathrm{MR}, \mathrm{BA}$, and $\mathrm{SR}$ contributed in paper revision.
13. Mabood F, Ibrahim SM, Khan WA. Effect of melting and heat generation/absorption on Sisko nanofluid over a stretching surface with nonlinear radiation. Phys Scr. (2019) 94:e065701. doi: 10.1088/1402-4896/ab1164

14. Javed M, Alderremy AA, Farooq M, Anjum A, Ahmad S, Malik MY. Analysis of activation energy and melting heat transfer in MHD flow with chemical reaction. Eur Phys J Plus. (2019) 134:256. doi: 10.1140/epjp/i201912744-5

15. Hayat T, Muhammad K, Alsaedi A, Ahmed B. Melting effect in squeezing flow of third-garde fluid with non-Fourier heat flux model. Phys Scr. (2019) 94:e105705. doi: 10.1088/1402-4896/ab1c2c

16. Hayat T, Shah F, Alsaedi A, Ahmad B. Entropy optimized dissipative flow of effective Prandtl number with melting heat transport and Joule heating. Int Commun Heat Mass Transf. (2020) 111:e104454. doi: 10.1016/j.icheatmasstransfer.2019.104454

17. Dinh MT, Tlili I, Dara RN, Shafee A, Al-Jahmany YY, NguyenThoi T. Nanomaterial treatment due to imposing MHD flow considering melting surface heat transfer. Physica A. (2020) 541:e123036. doi: 10.1016/j.physa.2019.123036

18. Hajipour M, Jajarmi A, Malek A, Baleanu D. Positivity-preserving sixthorder implicit finite difference weighted essentially non-oscillatory scheme for the nonlinear heat equation. Appl Math Comput. (2018) 325:14658. doi: 10.1016/j.amc.2017.12.026

19. Hajipour M, Jajarmi A, Baleanu D, Sun H. On an accurate discretization of a variable-order fractional reaction-diffusion equation. Commun Nonlinear Sci Numerical Simul. (2019) 69:119-33. doi: 10.1016/j.cnsns.2018. 09.004

20. Liao SJ. Homotopy Analysis Method in Non-linear Differential Equations. Heidelberg: Springer and Higher Education Press (2012).

21. Turkyilmazoglu M. An effective approach for evaluation of the optimal convergence control parameter in the homotopy analysis method. Filomat. (2016) 30:1633-50. doi: 10.2298/FIL1606633T

22. Muhammad T, Hayat T, Alsaedi A, Qayyum A. Hydromagnetic unsteady squeezing flow of Jeffrey fluid between two parallel plates. Chin J Phys. (2017) 55:1511-22. doi: 10.1016/j.cjph.2017.05.008

23. Hayat T, Rashid M, Khan MI, Alsaedi A. Melting heat transfer and induced magnetic field effects on flow of water based nanofluid over a rotating disk with variable thickness. Results Phys. (2018) 9:161830. doi: 10.1016/j.rinp.2018.04.054

24. Farooq M, Ahmad S, Javed M, Anjum A. Melting heat transfer in squeezed nanofluid flow through Darcy Forchheimer medium. J Heat Transf. (2019) 141:e012402. doi: 10.1115/1.40 41497 
25. Baleanu D, Asad JH, Jajarmi A. New aspects of the motion of a particle in a circular cavity. Proc Rom Acad Ser A. (2018)19:361-7. Available online at: https://scholar.ptuk.edu.ps/handle/123456789/625

26. Jajarmi A, Baleanu D, Sajjadi SS, Asad JH. A new feature of the fractional euler-lagrange equations for a coupled oscillator using a nonsingular operator approach. Front Phys. (2019) 7:196. doi: 10.3389/fphy.2019.00196

27. Baleanu D, Sajjadi SS, Jajarmi A, Asad JH. New features of the fractional Euler-Lagrange equations for a physical system within non-singular derivative operator. Eur Phys J Plus. (2019) 134:181. doi: 10.1140/epjp/i2019-12561-x

28. Baleanu D, Jajarmi A, Asad JH. The fractional model of spring pendulum: new features within different kernels. Proc Rom Acad Ser A. (2018) 19:447-54. Available online at: https://scholar.ptuk.edu.ps/handle/123456789/626

29. Waqas M, Shehzad SA, Hayat T, Khan MI, Alsaedi A. Simulation of magnetohydrodynamics and radiative heat transport in convectively heated stratified flow of Jeffrey nanofluid. J Phys Chem Solids. (2019) 133:4551. doi: 10.1016/j.jpcs.2019.03.031

Conflict of Interest: The authors declare that the research was conducted in the absence of any commercial or financial relationships that could be construed as a potential conflict of interest.

Copyright (c) 2020 Ahmad, Farooq, Rizwan, Ahmad and Rehman. This is an openaccess article distributed under the terms of the Creative Commons Attribution License (CC BY). The use, distribution or reproduction in other forums is permitted, provided the original author(s) and the copyright owner(s) are credited and that the original publication in this journal is cited, in accordance with accepted academic practice. No use, distribution or reproduction is permitted which does not comply with these terms. 